\title{
A MIMO System with Backward Compatibility for OFDM-Based WLANs
}

\author{
Jianhua Liu \\ Department of Electrical and Computer Engineering, University of Florida, P.O. Box 116130, \\ Gainesville, FL 32611-6130, USA \\ Email: jhliu@dsp.ufl.edu \\ Jian Li \\ Department of Electrical and Computer Engineering, University of Florida, P.O. Box 116130, \\ Gainesville, FL 32611-6130, USA \\ Email:li@dsp.ufl.edu
}

Received 16 December 2002; Revised 28 June 2003

\begin{abstract}
Orthogonal frequency division multiplexing (OFDM) has been selected as the basis for the new IEEE 802.11a standard for highspeed wireless local area networks (WLANs). We consider doubling the transmission data rate of the IEEE 802.11a system by using two transmit and two receive antennas. We propose a preamble design for this multi-input multi-output (MIMO) system that is backward compatible with its single-input single-output (SISO) counterpart as specified by the IEEE 802.11a standard. Based on this preamble design, we devise a sequential method for the estimation of the carrier frequency offset (CFO), symbol timing, and MIMO channel response. We also provide a simple soft detector based on the unstructured least square approach to obtain the soft information for the Viterbi decoder. This soft detector is very simple since it decouples the multidimensional QAM symbol detection into multiple one-dimensional QAM symbol—and further PAM symbol-detections. Both the sequential parameter estimation method and the soft detector can provide excellent overall system performance and are ideally suited for real-time implementations. The effectiveness of our methods is demonstrated via numerical examples.
\end{abstract}

Keywords and phrases: MIMO system, OFDM, WLAN, symbol timing, carrier synchronization, channel estimation.

\section{INTRODUCTION}

Orthogonal frequency division multiplexing (OFDM) has been selected as the basis for several new high-speed wireless local area network (WLAN) standards [1], including IEEE 802.11a [2], IEEE 802.11g, and HIPERLAN/2. IEEE 802.11g and HIPERLAN/2 are very similar to IEEE 802.11 a in terms of signal generation and detection/decoding. We use IEEE 802.11a to exemplify our presentation in this paper.

The OFDM-based WLAN system, as specified by the IEEE 802.11a standard, uses packet-based transmission. Each packet, as shown in Figure 1, consists of an OFDM packet preamble, a signal field, and an OFDM data field. The preamble can be used to estimate the channel parameters such as the carrier frequency offset (CFO), symbol timing, as well as channel response. These parameters are needed for the data symbol detection in the OFDM data field. The preamble design adopted by the standard is specifically tailored to the single-input single-output (SISO) system case where both the transmitter and receiver deal with a single signal. This standard supports a data rate up to $54 \mathrm{Mbps}$.
Transmission data rates higher than 54 Mbps are of particular importance for future WLANs. Deploying multiple antennas at both the transmitter and receiver is a promising way to achieve a high transmission data rate for multipathrich wireless channels without increasing the total transmission power or bandwidth [3]. The corresponding system, as shown in Figure 2, is referred to as a multi-input multioutput (MIMO) wireless communication system, where $M$ and $N$ in the figure denote the numbers of transmit and receive antennas, respectively.

Among the various popular MIMO wireless communication schemes, the BLAST (Bell Labs Layered Space Time) approaches $[4,5]$ are particularly attractive. BLAST attempts to achieve the potentially large channel capacity offered by the MIMO system $[6,7]$. In BLAST systems, the data stream is demultiplexed into independent substreams that are referred to as layers. These layers are transmitted simultaneously, that is, one layer per transmit antenna. At the receiver, the multiple layers can be detected, for example, through successive detection via an interference cancellation and nulling algorithm (ICNA) [5]. The detection 


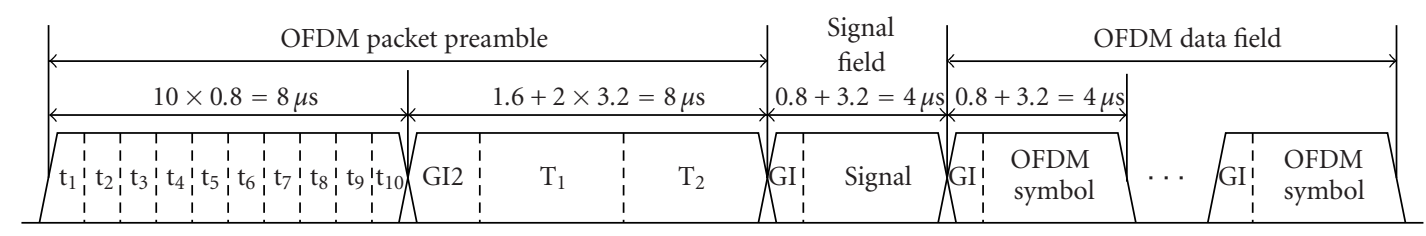

FIGURE 1: Packet structure of the IEEE 802.11a standard.

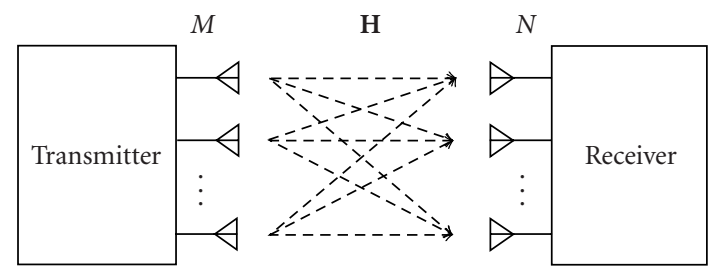

FIgURE 2: Diagram of a MIMO system.

can also be done via the sphere decoding (SPD) algorithm [8].

Our focus herein is on doubling the data rate of the SISO system as specified by the IEEE 802.11a standard by using two transmit and two receive antennas (referred to as the MIMO system in the sequel) based on the BLAST scheme. We propose a preamble design for this MIMO system that is backward compatible with its SISO counterpart as specified by the IEEE 802.11a standard. That is, a SISO receiver can perform CFO, symbol timing, and channel response estimation based on the proposed preamble design and detect up to the signal field. The SISO receiver is then informed, by using, for example, the reserved bit in the signal field, that a transmission is a SISO or not. Our preamble design can be used with two transmit and any number of receive antennas. However, we mainly focus on the two receive antenna case herein. Based on our MIMO preamble design, we propose a sequential method, ideally suited for real-time implementations, to estimate the CFO, symbol timing, and MIMO channel response.

The convolutional code specified in the IEEE 802.11a standard will also be used in our MIMO system for channel coding. As a result, soft information from the MIMO detector is needed by the Viterbi decoder to improve the decoding performance. Both the efficient ICNA and SPD algorithms offer only hard output. Soft output can be inferred with the ICNA-based algorithm for iterative detection and decoding [9]. However, this algorithm is computationally extremely heavy-exponentional in terms of transmit antenna number $M$ as well as the constellation size. Although reduced complexity versions were alluded to in [9], the costs in performance degradation by using these versions were not clear. The space time bit-interleaved coded modulation (STBICM) approach [10] can deliver soft output, in both the iterative and noniterative modes, but it is also computationally extremely heavy. A list sphere decoder (LSD) algorithm [11] was recently proposed to reduce the computational complex- ity of STBICM with a small performance degradation. However, LSD is still very complicated and hard to implement in real time for OFDM-based MIMO WLAN applications due to the high data rate. We present herein a simple MIMO soft detector, based on the unstructured least square (LS) fitting approach. This LS-based soft detector is ideally suited for real-time implementations since it decouples the multidimensional quadrature amplitude modulation (QAM) symbol detection into multiple one-dimensional QAM symbol detections. We show that the real and imaginary parts of the noise of the decoupled detection output are independent of each other. Hence, the QAM symbol detection can be further simplified into two pulse amplitude modulation (PAM) symbol detections. As a result, this LS-based soft detector is orders of magnitude more computationally efficient than LSD; yet, the efficiency is achieved at a cost of a small performance degradation, due to the aforementioned decoupling. The LS-based detector can also be seen as related to the zero-forcing or to the linear decorrelating detector [12].

The remainder of this paper is organized as follows. Section 2 describes the MIMO system. The new preamble design is given in this section. Section 3 presents our sequential method for CFO, symbol timing, and MIMO channel response estimation. The MIMO soft detector is provided in Section 4. Numerical examples are given in Section 5 to demonstrate the effectiveness of the proposed methods. Finally, we end our paper with comments and conclusions in Section 6.

\section{SYSTEM DESIGN}

Our MIMO system closely resembles its SISO counterpart as specified by the IEEE 802.11a standard. We first give a brief overview of the IEEE 802.11a SISO system before we proceed to describe our MIMO system.

\subsection{IEEE 802.11a standard}

Figure 1 shows the packet structure as specified by the IEEE 802.11a standard. The nominal bandwidth of the OFDM signal is $20 \mathrm{MHz}$ and the in-phase/quadrature (I/Q) sampling interval $t_{S}$ is 50 nanoseconds. In this case, the number of samples $N_{S}=64$ for an OFDM data symbol is equal to the number of subcarriers. The OFDM packet preamble consists of ten identical short OFDM training symbols $t_{i}$, $i=1,2, \ldots, 10$, each of which contains $N_{C}=16$ samples, and two identical long OFDM training symbols $\mathrm{T}_{i}, i=1,2$, each of which contains $N_{S}=64$ samples. Between the short and 
long OFDM training symbols, there is a long guard interval (GI2) consisting of $2 N_{C}=32$ data samples. GI2 is the cyclic prefix $(\mathrm{CP})$ for the long OFDM training symbol $\mathrm{T}_{1}$, that is, it is the exact replica of the last $2 N_{C}$ samples of $T_{1}$.

The information carrying data are encoded in the OFDM data field. The binary source data sequence is first scrambled and then convolutionally encoded by an industrial standard constraint length $K=7$, rate $1 / 2$ encoder, which has generation polynomials $g_{0}=(133)_{8}$ and $g_{1}=(171)_{8}$. The encoded output is then punctured according to the data rate requirement and is segmented into blocks of length $N_{\mathrm{CBPS}}$ (number of coded bits per OFDM symbol), each of which corresponds to an OFDM data symbol. The binary data in each block is first interleaved among the subcarriers (referred to as the frequency domain (FD) interleaving in the sequel) and then mapped (in groups of $\log _{2} A$ bits) into $A$-QAM symbols, which are used to modulate the different data carrying subcarriers. Each OFDM data symbol in the OFDM data field employs $N_{S}=64$ subcarriers, 48 of which are used for data symbols and 4 for pilot symbols. There are also 12 null subcarriers with one in the center and the other 11 on the two ends of the frequency band. The OFDM data symbols, each of which consists of $N_{S}=64$ samples, are obtained via taking the inverse fast Fourier transform (IFFT) of the data symbols, pilot symbols, and nulls on these $N_{S}$ subcarriers. To eliminate the intersymbol interference (ISI), each OFDM data symbol is preceded by a CP or GI, which contains the last $N_{C}$ samples of the OFDM data symbol.

The signal field contains the information including the transmission data rate and data length of the packet. The information is contained in 16 binary bits. There is also a reserved bit (which can be used to distinguish the MIMO from SISO transmissions) and a parity check bit. These 18 bits, padded with 6 zeros, are then encoded (by the same encoder as for the OFDM data field) to obtain a 48-bit binary sequence. The encoded sequence is then interleaved among subcarriers and used to modulate the 48 data carrying subcarriers using BPSK. The signal field consists of 64 samples and is obtained via taking the IFFT of these 48 BPSK symbols, 4 pilot symbols, and 12 nulls. Also, there is a CP of length $N_{C}$ to separate the preamble from the signal field.

\subsection{SISO data model}

To establish the data model, consider first the generation of an OFDM data symbol in the OFDM data field. Let $\mathbf{x}_{\text {SISO }}=\left[\begin{array}{lllll}x_{1}^{1} & x_{2}^{1} & \cdots & x_{N_{S}}^{1}\end{array}\right]^{T}$ be a vector of $N_{S}$ data symbols, where $(\cdot)^{T}$ denotes the transpose and $x_{n_{S}}^{1}, n_{S}=1,2, \ldots, N_{S}$, is the symbol modulating the $n_{S}$ th subcarrier and is equal to 0 for null subcarriers, 1 or -1 for pilot subcarriers, and in $\mathcal{C}$ for data carrying subcarriers. Here $\mathcal{C}$ is a finite constellation, such as BPSK, QPSK, 16-QAM, or 64-QAM. Let $\mathbf{W}_{N_{S}} \in \mathbb{C}^{N_{S} \times N_{S}}$ be the fast Fourier transform (FFT) matrix. Then the OFDM data symbol $\mathbf{s}$ corresponding to $\mathbf{x}_{\mathrm{SISO}}$ is obtained by taking the IFFT of $\mathbf{x}_{\mathrm{SISO}}$. That is, $\mathbf{s}=\mathbf{W}_{N_{S}}^{H} \mathbf{x}_{\mathrm{SISO}} / N_{S}$, where $(\cdot)^{H}$ denotes the conjugate transpose. To eliminate the ISI, each OFDM data symbol is preceded by a CP or GI $\mathbf{s}_{C}$ formed using $\mathbf{s}$.
Let

$$
h^{(\mathrm{t})}(t)=\sum_{p} \alpha_{p} \delta\left(t-\tau_{p} t_{S}\right)
$$

denote the time-domain analogue channel impulse response of the frequency-selective time-invariant fading channel, where $\alpha_{p}$ and $\tau_{p} t_{S}, 0 \leq \tau_{p} \leq N_{C}, p \in \mathbb{Z}$, are the complex gain and time delay of the $p$ th path, respectively. Let

$$
\mathbf{h}^{(\mathrm{t})}=\left[\begin{array}{llll}
h_{0}^{(\mathrm{t})} & h_{1}^{(\mathrm{t})} & \cdots & h_{N_{S}-1}^{(\mathrm{t})}
\end{array}\right]^{T}
$$

be the equivalent finite impulse response (FIR) filter response of $h^{(\mathrm{t})}(t)$, that is, if $\mathbf{h}=\mathbf{W}_{N_{S}} \mathbf{h}^{(\mathrm{t})}=\left[\begin{array}{llll}h_{1} & h_{2} & \cdots & h_{N_{S}}\end{array}\right]^{T}$ is the sampled frequency domain channel response, then for $n_{S}=1,2, \ldots, N_{S}$,

$$
h_{n_{S}}=\left.\sum_{p} \alpha_{p} e^{-j \tau_{p} t_{S} \omega}\right|_{\omega=2 \pi\left(n_{S}-1\right) /\left(N_{S} t_{S}\right)}
$$

The $l$ th element of $\mathbf{h}^{(\mathrm{t})}, l=0,1, \ldots, N_{S}-1$, can be written as

$$
h_{l}^{(\mathrm{t})}=\sum_{p} \alpha_{p} e^{-j \pi\left(l+\left(N_{S}-1\right) \tau_{p}\right) / N_{S}} \frac{\sin \left(\pi \tau_{p}\right)}{\sin \left(\pi\left(\tau_{p}-l\right) / N_{S}\right)},
$$

which includes the leakage effect due to the frequency domain sampling [13].

By discarding the first $N_{C}$ samples at the receiver (assuming a correct symbol timing), the noise-free and $\mathrm{CFO}$ free received signal vector $\mathbf{z}_{\text {SISO }}^{\text {ne }} \in \mathbb{C}^{N_{S} \times 1}$, due to sampling the received signal, is the circular convolution of $\mathbf{h}^{(t)}$ and $\mathbf{s}$. Hence the FFT output of the received data vector $\mathbf{z}_{\text {SISO }}=$ $\mathbf{z}_{\text {SISO }}^{\text {ne }}+\mathbf{e}_{\text {SISO }}$, where $\mathbf{e}_{\text {SISO }} \sim \mathcal{N}\left(\mathbf{0},\left(\sigma^{2} / N_{S}\right) \mathbf{I}_{N_{S}}\right)$ is the additive zero-mean white circularly symmetric complex Gaussian noise with variance $\sigma^{2}$, can be written as [14]

$$
\mathbf{y}_{\text {SISO }}=\mathbf{W}_{N_{S}} \mathbf{z}_{\text {SISO }}=\operatorname{diag}\{\mathbf{h}\} \mathbf{x}_{\text {SISO }}+\mathbf{W}_{N_{S}} \mathbf{e}_{\text {SISO }} \in \mathbb{C}^{N_{S} \times 1} \text {. }
$$

The data model in (5) can also represent the OFDM symbols in the signal field and the preamble.

Equation (5) can also be written as

$$
\mathbf{y}_{\text {SISO }}=\operatorname{diag}\left\{\mathbf{x}_{\text {SISO }}\right\} \mathbf{h}+\mathbf{W}_{N_{S}} \mathbf{e}_{\text {SISO }}
$$

Note that (5) is useful for symbol detection whereas (6) is used for channel estimation.

For the sake of simulation simplicity, the equivalent channel $\mathbf{h}^{(t)}$ is often approximated by an exponentially decaying FIR filter with length $L_{F}$ [14], denoted as

$$
\mathbf{h}_{L_{F}}^{(\mathrm{t})}=\left[\begin{array}{llll}
h_{0}^{(\mathrm{t})} & h_{1}^{(\mathrm{t})} & \cdots & h_{L_{F}-1}^{(\mathrm{t})}
\end{array}\right]^{T} .
$$

In this case, the received signal can be easily simulated as the convolution of the channel $\mathbf{h}_{L_{F}}^{(\mathrm{t})}$ and the transmitted signal. Let $t_{r}$ be the root mean square (RMS) delay spreading time and $t_{n}=t_{r} / t_{S}$. Then $L_{F}=\left\lceil 10 t_{n}\right\rceil+1$, where $\lceil x\rceil$ denotes the smallest integer not less than $x$. For $l_{F}=0,1, \ldots, L_{F}-1$, we have

$$
h_{l_{F}}^{(\mathrm{t})} \sim \mathcal{N}\left(0,\left(1-e^{-1 / t_{n}}\right) e^{-l_{F} / t_{n}}\right) .
$$

This channel model is referred to as the Chayat model [15]. 


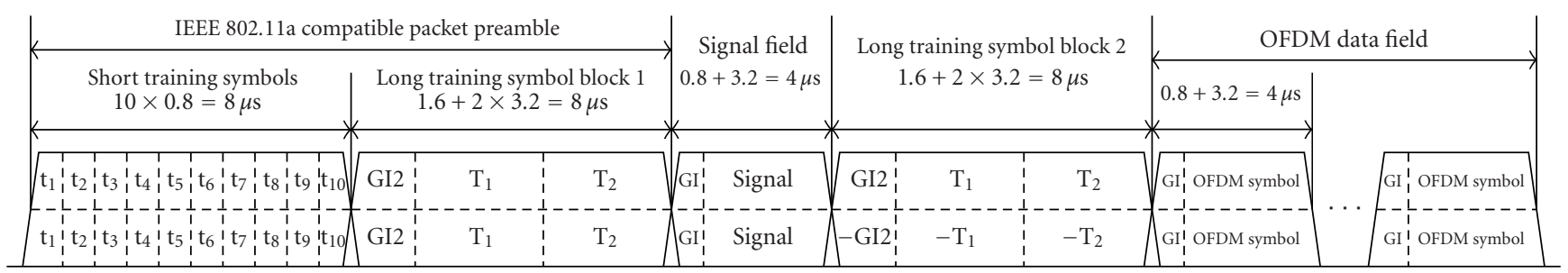

Figure 3: Proposed MIMO preamble (and signal field) structure.

Note that our symbol timing estimation method, which will be presented in Section 3, works equally well for the channel models given by both (4) and (7). Our MIMO channel response estimation method also works equally well for both models. We use (7) to generate channels to simplify our simulations.

\subsection{MIMO preamble design}

For the IEEE 802.11a SISO system, the short OFDM training symbols can be used to detect the arrival of the packet, allow the automatic gain control (AGC) to stabilize, compute a coarse CFO estimate, and obtain a coarse symbol timing, whereas the long OFDM training symbols can be used to calculate a fine $\mathrm{CFO}$ estimate, refine the coarse symbol timing, and estimate the SISO channel response.

The MIMO system considered herein has two transmit and two receive antennas (such as a crossed dipole pair for both the transmitter and the receiver). Two packets are transmitted simultaneously from the two transmit antennas. We design two preambles, one for each transmit antenna. We assume that the receiver antenna outputs suffer from the same $\mathrm{CFO}$ and has the same symbol timing. To be backward compatible with the SISO system, we use the same short OFDM training symbols as in the SISO preamble for both of the MIMO transmit antennas, as shown in Figure 3.

As for the long OFDM training symbols, they should be designed to support the MIMO channel response estimation. MIMO channel response estimation has attracted much research interest lately. Orthogonal training sequences tend to give the best performance (see, e.g., [16] and the references therein). We also adopt this idea of orthogonal training sequences in our preamble design. In the interest of backward compatibility, we use the same $\mathrm{T}_{1}$ and $\mathrm{T}_{2}$ (as well as GI2) as for the SISO system for both of the MIMO transmit antennas before the signal field, as shown in Figure 3. After the signal field, we use $T_{1}$ and $T_{2}$ (and GI2) for one transmit antenna, and $-\mathrm{T}_{1}$ and $-\mathrm{T}_{2}$ (and - GI2) for the other. This way, when the simultaneously transmitted packets are received by a single SISO receiver, the SISO receiver can successfully detect up to the signal field, which is designed to be the same for both transmit antennas. The reserved bit in the signal field can tell the SISO receiver to stop its operation whenever a MIMO transmission follows or otherwise to continue its operation. The long OFDM training symbols before and after the signal field are used in the MIMO receivers for channel estimation. Although the employment of an additional pair of long OFDM training symbols can increase the overhead, the corresponding loss of efficiency is not significant for larger packet. The reserved bit in the signal field can also inform the MIMO receiver that the transmission is a SISO one. When this occurs, the MIMO receiver can modify its channel estimation and the data bit detection steps slightly, as detailed at the end of Sections 3 and 4, respectively.

Other MIMO preamble design options with backward compatibility are possible. For example, by exploiting the transmit/receive diversities, we may get improved symbol timing or $\mathrm{CFO}$ correction. However, these improvements do not necessarily result in improved packet error rate (PER). Hence, we prefer the straightforward MIMO preamble design shown in Figure 3.

\subsection{MIMO data model}

To stay as close to the IEEE 802.11a standard as possible, we use in our MIMO system the same scrambler, convolutional encoder, puncturer, FD interleaver, symbol mapper, pilot sequence, and $\mathrm{CP}$ as specified in the standard. To improve diversity, we add a simple spatial interleaver to scatter every two consecutive bits across the two transmit antennas. This spatial interleaving is performed before the FD interleaving.

Consider the $n_{S}$ th subcarrier (for notational convenience, we drop the notational dependence on $n_{S}$ below). Consider the case of $N$ receive antennas. (Note that considering the general case of $N$ receive antennas does not add extra difficulties for the discussions below.) Let $\mathbf{H}$ denote the MIMO channel matrix for the $n_{S}$ th subcarrier:

$$
\mathbf{H}=\left[\begin{array}{cc}
h_{1,1} & h_{1,2} \\
h_{2,1} & h_{2,2} \\
\vdots & \vdots \\
h_{N, 1} & h_{N, 2}
\end{array}\right] \in \mathbb{C}^{N \times 2},
$$

where $h_{n, m}$ denotes the channel gain from the $m$ th transmit antenna to the $n$th receive antenna for the $n_{S}$ th subcarrier. Let $\mathbf{y}$ denote a received data vector for the $n_{S}$ th subcarrier, which can be written as

$$
\mathbf{y}=\mathbf{H x}+\mathbf{e} \in \mathbb{C}^{N \times 1},
$$

where $\mathbf{x}=\left[\begin{array}{ll}x_{1} & x_{2}\end{array}\right]^{T}$ is the $2 \times 1$ QAM symbol vector sent on the $n_{S}$ th subcarrier and $\mathbf{e} \sim \mathcal{N}\left(\mathbf{0}, \sigma^{2} \mathbf{I}_{N}\right)$ is the additive white circularly symmetric complex Gaussian noise with variance $\sigma^{2}$. In Section 4, we will provide a soft detector based on this model. 


\section{CFO, SYMBOL TIMING, AND CHANNEL ESTIMATION}

In this section, we present our sequential CFO, symbol timing, and MIMO channel response estimation approach based on our MIMO preamble design. The CFO can be estimated from the samples of two consecutive data blocks due to the periodic inputs (the short OFDM training symbols $\left.t_{1}, \ldots, t_{10}\right)$. Because of the fact that the CFO can be outside the unambiguous range measurable by the long OFDM training symbols, we have to estimate the CFO in two steps: (a) a coarse CFO estimation using the short OFDM training symbols and then (b) a fine CFO estimation, to determine the residue of the coarse CFO correction, using the long OFDM training symbols. After estimating and accounting for the CFO, we can obtain the symbol timing. We estimate the symbol timing also in two steps: the coarse symbol timing and fine symbol timing. The former is obtained by using the later portion of the short OFDM training symbols in the packet preamble. The fine symbol timing is obtained by using the long OFDM training symbols before the signal field. Finally, we obtain the MIMO channel response estimate. The parameter estimates are obtained in the order presented below.

\subsection{Coarse CFO estimation}

Let $z_{n}(l)=z_{n}^{\text {ne }}(l)+e_{n}(l), n=1, \ldots, N$, denote the $l$ th time sample of the signal received from the $n$th receive antenna, starting from the moment that the receiver AGC has become stationary (the receiver AGC is assumed to become stationary at least before receiving the last two short OFDM training symbols and remain stationary while receiving the remainder of the packet). In the presence of CFO, we have [17]

$$
z_{n}^{\text {ne }}\left(l+N_{C}\right)=z_{n}^{\text {ne }}(l) e^{j 2 N_{C} \pi \epsilon}, \quad n=1, \ldots, N
$$

where $\epsilon$ is the normalized CFO (with respect to the sampling frequency), which we still refer to as CFO for convenience. For each receive antenna output, consider the correlation between two consecutive noise-free received data blocks, each of which is of length $N_{C}$. Then the sum of the correlations for all receive antennas can be written as

$$
\begin{aligned}
\sum_{n=1}^{N} \sum_{l=k}^{k+N_{C}-1} z_{n}^{\mathrm{ne}}(l)\left(z_{n}^{\mathrm{ne}}\left(l+N_{C}\right)\right)^{*} & =e^{-j 2 N_{C} \pi \epsilon} \sum_{n=1}^{N} \sum_{l=0}^{N_{C}-1}\left|z_{n}^{\mathrm{ne}}(l)\right|^{2} \\
& \triangleq P e^{-j 2 N_{C} \pi \epsilon}
\end{aligned}
$$

where $(\cdot)^{*}$ denotes the complex conjugate and $k$ is any nonnegative integer such that $z_{n}^{\text {ne }}\left(k+2 N_{C}-1\right)$ is a sample of the $n$th receive antenna output due to the input (transmit antenna output) being a sample of the short OFDM training symbols of the MIMO packet preamble. Let

$$
\begin{aligned}
P_{S} & =\sum_{n=1}^{N} \sum_{l=0}^{N_{C}-1} z_{n}(l) z_{n}^{*}\left(l+N_{C}\right) \\
& =P e^{-j 2 N_{C} \pi \epsilon}+e_{P},
\end{aligned}
$$

where $e_{P}$ is due to the presence of the noise. We calculate the coarse $\mathrm{CFO}$ as [18]

$$
\hat{\epsilon}_{C}=-\frac{1}{2 N_{C} \pi} \angle P_{S},
$$

where $\angle x$ denotes taking the argument of $x$.

We next correct the CFO using $\hat{\epsilon}_{C}$ to get the data samples $z_{n}^{(C)}(l), n=1,2, \ldots, N$, as follows:

$$
z_{n}^{(C)}(l)=z_{n}(l) e^{-j 2 l \pi \hat{\epsilon}_{C}} .
$$

Correspondingly, we have

$$
P_{S}^{(C)}=P_{S} e^{j 2 N_{C} \pi \hat{\epsilon}_{C}}
$$

In the sequel, we only consider the CFO corrected data given above. For notational convenience, we drop the superscript of $z_{n}^{(C)}(l), n=1,2, \ldots, N$.

\subsection{Coarse symbol timing estimation}

Now we can use a correlation method, modified based on the approach presented in [17] to estimate the coarse symbol timing. The symbol timing is referred to as the starting time sample due to the input being the long OFDM training symbol $\mathrm{T}_{1}$ (before the signal field). Once the starting time sample due to the long OFDM training symbol $\mathrm{T}_{1}$ is determined, we can determine the starting time sample due to every OFDM data symbol thereafter. According to the specification of the IEEE 802.11a standard and the sampling rate of $20 \mathrm{MHz}$, the true symbol timing $T_{0}$ is 193 , as shown in Figure 4.

From (13) and (16), we note that the correlation (after the CFO correction) is approximately the real-valued scalar $P$ plus a complex-valued noise. Hence we propose to use the following real-valued correlation sequence for coarse symbol timing determination. We calculate the correlation sequence in an iterative form similar to the complex-valued approach in [17] as follows:

$$
\begin{aligned}
& P_{R}(k+1) \\
& =P_{R}(k) \\
& \quad+\operatorname{Re}\left\{\sum_{n=1}^{N}\left[z_{n}\left(k+N_{C}\right) z_{n}^{*}\left(k+2 N_{C}\right)-z_{n}(k) z_{n}^{*}\left(k+N_{C}\right)\right]\right\} \\
& =P_{R}(k)+\sum_{n=1}^{N}\left\{\bar{z}_{n}\left(k+N_{C}\right)\left[\bar{z}_{n}\left(k+2 N_{C}\right)-\bar{z}_{n}(k)\right]\right. \\
& \left.\quad+\tilde{z}_{n}\left(k+N_{C}\right)\left[\tilde{z}_{n}\left(k+2 N_{C}\right)-\tilde{z}_{n}(k)\right]\right\},
\end{aligned}
$$

where both $\operatorname{Re}(\cdot)$ and $\left(^{-}\right)$denote the real part of a complex entity and $\left({ }^{\circ}\right)$ stands for the imaginary part. We start the iteration by using $P_{R}(0)=\operatorname{Re}\left(P_{S}\right)$. Note that the real-valued correlation approach given in (17) is superior to the absolutevalued one given in [17] since the former uses fewer computations, lowers the noise level (variance reduced in half) in the correlation sequence, and decreases closer to zero when the data samples in the sliding data blocks are due to the 


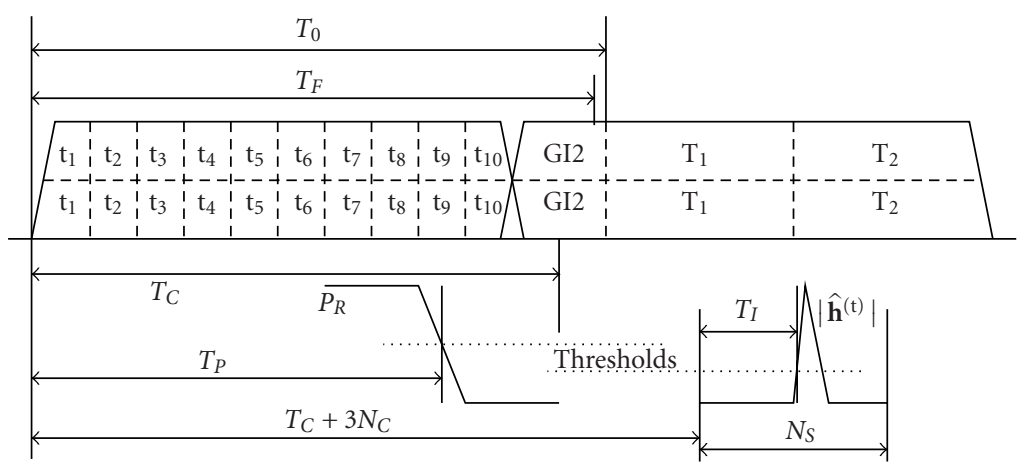

FIGURE 4: Illustration of symbol timing determination.

input being GI2 or the long OFDM training symbols following the short OFDM training symbols in the preamble.

When some of the data samples of the sliding data blocks are taken from the received data due to the input being GI2 or the long training symbols following the short OFDM training symbol, $P_{R}(k)$ will drop since (11) no longer holds. This property is used to obtain the coarse symbol timing. Let $T_{P}$, as shown in Figure 4, denote the first time sample when $P_{R}(k)$ drops to less than half of its peak value. The coarse symbol timing

$$
T_{C}=T_{P}+\frac{3}{2} N_{C}+N_{C}
$$

is the coarse estimate of the beginning time sample due to the input being the long OFDM training symbol $\mathrm{T}_{1}$ before the signal field. The second term at the right-hand side of the above equation is due to the fact that $P_{R}(k)$ will drop to approximately one half of its maximum value when the data samples of the second half of the second of the two sliding blocks are due to the first GI2 in the preamble as input; the third term is due to one half of the length of GI2. When the channel spreading delay $t_{D}=\max \left\{\tau_{p}-\tau_{l}\right\}$ is assumed to satisfy $t_{D} \leq N_{C}$, only the first half of GI2 can suffer from ISI. Hence our goal of coarse timing determination is to place the coarse timing estimate between the true timing $T_{0}=193$ and $T_{0}-N_{C}=177$ to make accurate fine CFO estimation possible. This explains why we use $N_{C}$ instead of $2 N_{C}$ for the third term in (18).

\subsection{Fine CFO estimation}

For each receive antenna output, we calculate the correlation between the two long OFDM training symbols before the signal field. We then sum the correlations for all receive antennas as follows:

$$
P_{L}=\sum_{n=1}^{N} \sum_{l=0}^{N_{S}-1} z_{n}\left(l+T_{C}\right) z_{n}^{*}\left(l+T_{C}+N_{S}\right) .
$$

Then the fine CFO estimate can be computed as

$$
\hat{\epsilon}_{F}=-\frac{1}{2 N_{S} \pi} \angle P_{L}
$$

We can use $\hat{\epsilon}_{F}$ in the same way as $\hat{\epsilon}_{C}$ to correct the CFO. We assume that for the data we use below, $\hat{\epsilon}_{F}$ has been already corrected.

Note that the aforementioned simple fine CFO estimation approach may not be optimal. For example, the CFO estimation accuracy could be improved by using the long OFDM training symbols after the signal field as well; however, our simple fine CFO estimation approach is sufficiently accurate in that the overall system performance can no longer be improved with a more accurate CFO estimate, especially when pilot symbols are exploited. For the data bit detection, no matter how accurate the CFO estimate is, it can never be perfect due to the presence of noise. Pilot symbols are used to track the CFO residual phase for each OFDM data symbol before data bit detection. A maximum likelihood (ML) CFO residual tracking scheme is given in Appendix C.

\subsection{Fine symbol timing estimation}

We now move on to obtain the fine symbol timing by using the long OFDM training symbols before the signal field. The fine symbol timing is estimated by using the $N$ data blocks of length $N_{S}$, starting from the time sample $T_{C}+3 N_{C}$. With this choice, due to the fact that $T_{1}$ is identical to $T_{2}$, the data blocks are most likely due to the input being the second half of $\mathrm{T}_{1}$ and the first half of $\mathrm{T}_{2}$, even when the coarse symbol timing has a large error.

Let $\mathbf{y}_{n}$ denote the $N_{S}$-point FFT of the data block from the $n$th receive antenna and let $\mathbf{h}_{n, m}^{(\mathrm{t})}$ be the equivalent FIR channel in the time domain between the $m$ th transmit antenna and the $n$th receive antenna, $n=1,2, \ldots, N, m=1,2$. Then, by neglecting the existence of the residual CFO, $\mathbf{y}_{n}$ can be written as (cf. (6))

$$
\mathbf{y}_{n}=\mathbf{X}_{B} \mathbf{W}_{N_{S}} \sum_{m=1}^{2} \mathbf{h}_{n, m}^{(\mathrm{t})}+\mathbf{W}_{N_{S}} \mathbf{e}_{n},
$$

where $\mathbf{X}_{B}$ is a diagonal matrix with the 52 known BPSK symbols and 12 zeros, which form the $\mathrm{T}_{1}$ in Figure 3 , on the diagonal. Since the Moore-Penrose pseudoinverse of $\mathbf{X}_{B}$ is $\mathbf{X}_{B}$ itself and $\mathbf{W}_{N_{S}} / N_{S}^{1 / 2}$ is unitary, we get an estimate of $\mathbf{h}_{n}^{(\mathrm{t})}=\sum_{m=1}^{2} \mathbf{h}_{n, m}^{(\mathrm{t})}$ as 


$$
\hat{\mathbf{h}}_{n}^{(\mathrm{t})}=\frac{1}{N_{S}} \mathbf{W}_{N_{S}}^{H} \mathbf{X}_{B} \mathbf{y}_{n}
$$

Let $T_{I}$, as shown in Figure 4, denote the index of the first element of $\left|\hat{\mathbf{h}}^{(\mathrm{t})}\right|=\sum_{n=1}^{N}\left|\hat{\mathbf{h}}_{n}^{(\mathrm{t})}\right|$ that is above $1 / 3$ of the maximum value of the elements of $\sum_{n=1}^{N}\left|\hat{\mathbf{h}}_{n}^{(\mathrm{t})}\right|$. (Our empirical experience suggests that selecting the threshold to be $1 / 3$ gives the best result.) Then the fine symbol timing $T_{F}$ is obtained as

$$
T_{F}=T_{C}-N_{C}+T_{I}-3 .
$$

The second term above is used to compensate for the aforementioned $3 N_{C}$ shift due to the fact that $N_{S}-3 N_{C}=N_{C}$ and the last term above is chosen to be 3 to ensure that $T_{F}>T_{0}$ occurs with very low probability.

\subsection{MIMO channel response estimation}

After we obtained $T_{F}$, we can now estimate the MIMO channel response. Let $\mathbf{y}_{n, 1}$ denote the $N_{S}$-point FFT of the average of the two consecutive blocks, each of which is of length $N_{S}$, associated with the two long training symbols before the signal field, from the $n$th receive antenna. Let $\mathbf{y}_{n, 2}$ denote the counterpart of $\mathbf{y}_{n, 1}$ after the signal field. Then, for the $n_{S}$ th subcarrier, we have

$$
\begin{aligned}
& y_{n, 1} \approx x_{B}\left(h_{n, 1}+h_{n, 2}\right), \\
& y_{n, 2} \approx x_{B}\left(h_{n, 1}-h_{n, 2}\right),
\end{aligned}
$$

where $x_{B}$ denotes the $n_{S}$ th diagonal element of $\mathbf{X}_{B}, y_{n, i}$ denotes the $n_{S}$ th element of $\mathbf{y}_{n, i}, i=1,2$, and we have dropped the dependence on $n_{S}$ for notational simplicity. Solving (24) and (25) yields

$$
\begin{aligned}
& \hat{h}_{n, 1}=\frac{x_{B}\left(y_{n, 1}+y_{n, 2}\right)}{2}, \\
& \hat{h}_{n, 2}=\frac{x_{B}\left(y_{n, 1}-y_{n, 2}\right)}{2} .
\end{aligned}
$$

When the reserved bit in the signal field indicates a SISO transmission, we only need to estimate $h_{n, 1}, n=1,2, \ldots, N$, in a way similar to (26).

\section{A SIMPLE MIMO SOFT DETECTOR}

With the CFO, symbol timing, and MIMO channel response determined and accounted for, we can proceed to detect the data bits contained in each BLAST layer and subcarrier of the OFDM data symbols in the OFDM data field. In the sequel, we present a very simple soft detector for the MIMO system. Note that this soft detector can be used in a general setting of the BLAST system and hence we present it in a general framework based on the data model of (10), where $\mathbf{H}$ is assumed to be $N \times M$ and $\mathbf{x}$ to be $M \times 1$. (We use $\hat{\mathbf{H}}$ to replace $\mathbf{H}$ in our simulations.)

Consider first the ML hard detector of the BLAST system. For the data model of (10), the ML hard detector is given by

$$
\hat{\mathbf{x}}=\arg \min _{\mathbf{x} \in \mathfrak{C}^{M \times 1}}\|\mathbf{y}-\mathbf{H x}\|^{2},
$$

where $\|\cdot\|^{2}$ denotes the Euclidean norm. The cost function in (28) can be written as

$$
\begin{aligned}
\|\mathbf{y}-\mathbf{H} \mathbf{x}\|^{2}= & \mathbf{y}^{H} \mathbf{y}+\mathbf{x}^{H} \mathbf{H}^{H} \mathbf{H} \mathbf{x}-\mathbf{y}^{H} \mathbf{H} \mathbf{x}-\mathbf{x}^{H} \mathbf{H}^{H} \mathbf{y} \\
= & \left(\mathbf{x}^{H}-\mathbf{y}^{H}\left(\mathbf{H}^{\dagger}\right)^{H}\right) \mathbf{H}^{H} \mathbf{H}\left(\mathbf{x}-\mathbf{H}^{\dagger} \mathbf{y}\right) \\
& +\mathbf{y}^{H} \mathbf{y}-\mathbf{y}^{H}\left(\mathbf{H}^{\dagger}\right)^{H} \mathbf{H}^{H} \mathbf{H} \mathbf{H}^{\dagger} \mathbf{y}
\end{aligned}
$$

where $\mathbf{H}^{\dagger}=\left(\mathbf{H}^{H} \mathbf{H}\right)^{-1} \mathbf{H}^{H}$. We note, from the above equation, that by ignoring the constellation constraint on $\mathbf{x}$, we can obtain an unstructured LS estimate $\hat{\mathbf{x}}_{\mathrm{us}}$ of $\mathbf{x}$, which is given by

$$
\hat{\mathbf{x}}_{\mathrm{us}}=\mathbf{H}^{\dagger} \mathbf{y}=\mathbf{x}+\mathbf{H}^{\dagger} \mathbf{e} \triangleq \mathbf{x}+\mathbf{c} .
$$

Note that $\hat{\mathbf{x}}_{\mathrm{us}}$ is the soft decision statistic that we are interested in. We refer to this simple scheme of obtaining a soft decision statistic as the MIMO soft detection scheme. Note that a necessary condition for $\mathbf{H}^{H} \mathbf{H}$ to be nonsingular is $N \geq M$. Also note that $\mathbf{c}$ is still Gaussian with zero mean and covariance matrix

$$
\mathrm{E}\left[\mathbf{c c}^{H}\right]=\sigma^{2} \mathbf{H}^{\dagger}\left(\mathbf{H}^{\dagger}\right)^{H}=\sigma^{2}\left(\mathbf{H}^{H} \mathbf{H}\right)^{-1} .
$$

Due to the use of the interleaver and deinterleaver, the data bits contained in $\mathbf{x}$ are independent of each other. By ignoring the dependence among the elements of $\mathbf{c}$, we can consider only the marginal probability density function ( $\mathrm{pdf}$ ) for the elements $\hat{x}_{\mathrm{us}}(m), m=1,2, \ldots, M$, of $\hat{\mathbf{x}}_{\mathrm{us}}$. (Note that an approximation is made here, which can lead to performance degradation. However, the computation is greatly simplified by the approximation.) Let

$$
\mathbf{H}^{\dagger} \triangleq\left[\begin{array}{c}
\breve{\mathbf{h}}_{1}^{T} \\
\breve{\mathbf{h}}_{2}^{T} \\
\vdots \\
\breve{\mathbf{h}}_{M}^{T}
\end{array}\right] \in \mathbb{C}^{M \times N}
$$

Then the $m$ th element of $\mathbf{c}, m=1,2, \ldots, M$, can be written as

$$
c_{m}=\breve{\mathbf{h}}_{m}^{T} \mathbf{e}
$$

Obviously, $c_{m}$ is still Gaussian with zero mean and variance

$$
\sigma_{m}^{2}=\mathrm{E}\left[\left|c_{m}\right|^{2}\right]=\left\|\breve{\mathbf{h}}_{m}\right\|^{2} \sigma^{2}
$$

The estimate of the above noise variance $\sigma^{2}$ can be easily obtained via the difference of the two consecutive blocks of the $n$th receive antenna, from which we got $\mathbf{y}_{n, 1}$ (cf. (24)). Note that $\sigma_{m}^{2}$ along with $\hat{x}_{\mathrm{us}}^{(m)}$ provide the soft information for the $m$ th, $m=1,2, \ldots, M$, symbol in $\hat{\mathbf{x}}_{\mathrm{us}}$, needed by the Viterbi decoder. Note also that the noises corresponding to different layers have different variances which means that the symbols corresponding to different layers have different quality. This unbalanced layer quality is the reason why we have used a spatial interleaver before the FD interleaver. 
Note that for SISO systems we usually consider an ordinary QAM symbol as two PAM symbols (e.g., a 64-QAM symbol can be considered as two 8-ary PAM symbols) due to the orthogonality between the real and imaginary parts of a QAM symbol as well as the independence between the real and imaginary parts of the additive circularly symmetric Gaussian error. A bit metric computation scheme for PAM symbols is presented in Appendix A. In Appendix B, we show that the real and imaginary parts of $c_{m}$ are independent of each other. Hence we can significantly simplify the bit metric computations by exploiting these independencies.

The minimum mean square error (MMSE) detector is often deemed to be better than the LS-based one [12]. Although this can be true for the constant modulus constellations, such as PSK, it is not necessarily true for QAM symbols, as suggested by our simulations due to the different power levels of the QAM symbols. Hence, we do not provide an MMSE counterpart of the LS-based soft detector.

When the reserved bit in the signal field indicates a SISO transmission, the $\mathbf{H}$ in (28) is in fact a vector. Hence the $\hat{\mathbf{x}}_{\text {us }}$ in (30) and the $\mathrm{E}\left[\mathrm{cc}^{\mathrm{H}}\right]$ in (31) are scalars, and they are the soft information used as in the SISO system for data bit detection.

\section{NUMERICAL EXAMPLES}

In this section, we provide numerical examples to demonstrate the effectiveness and performance of our sequential estimation method for CFO, symbol timing, and MIMO channel response based on our MIMO preamble design as well as the simple MIMO soft detector.

In the IEEE 802.11a standard, the maximum transmission data rate is $54 \mathrm{Mbps}$; in this case, the 64-QAM constellation is used and the channel coding rate is $R=3 / 4$, which comes from puncturing the $R_{C}=1 / 2$ encoded sequence with the puncturing rate $R_{P}=2 / 3$. We consider doubling the maximum $54 \mathrm{Mbps}$ transmission data rate by using two transmit and two receive antennas, that is, $M=N=2$. In our simulations, each of the $M N=4$ time domain MIMO channels is generated according to the Chayat model; the 4 channels are independent of each other.

Due to the fact that 52 out of 64 subcarriers are used in the OFDM-based WLAN system, the signal-to-noise ratio (SNR) for the SISO system used in this paper is defined as $52 /\left(64 \sigma^{2}\right)$ for the constellations whose average energies are normalized to 1 . Whereas for the MIMO system, the SNR is defined as $52 /\left(128 \sigma^{2}\right)$ (i.e., we use the same total transmission power for the MIMO system as for its SISO counterpart).

We first provide a simulation example for symbol timing estimation. Two curves in Figure 5 show the $10^{4}$ Monte Carlo simulation results of the coarse symbol timing estimates for the Chayat channels with $t_{r}=25$ and $t_{r}=50$ nanoseconds, respectively, when $\mathrm{SNR}=10 \mathrm{~dB}$. Note that the coarse symbol timing estimates fall within the desired interval with a high probability. Note also that the adverse effect of the coarse symbol timing estimate being smaller than $T_{0}-N_{C}=177$ is usually not significant since, due to the exponentially decaying property of the channels, the ISI in the receiver out-

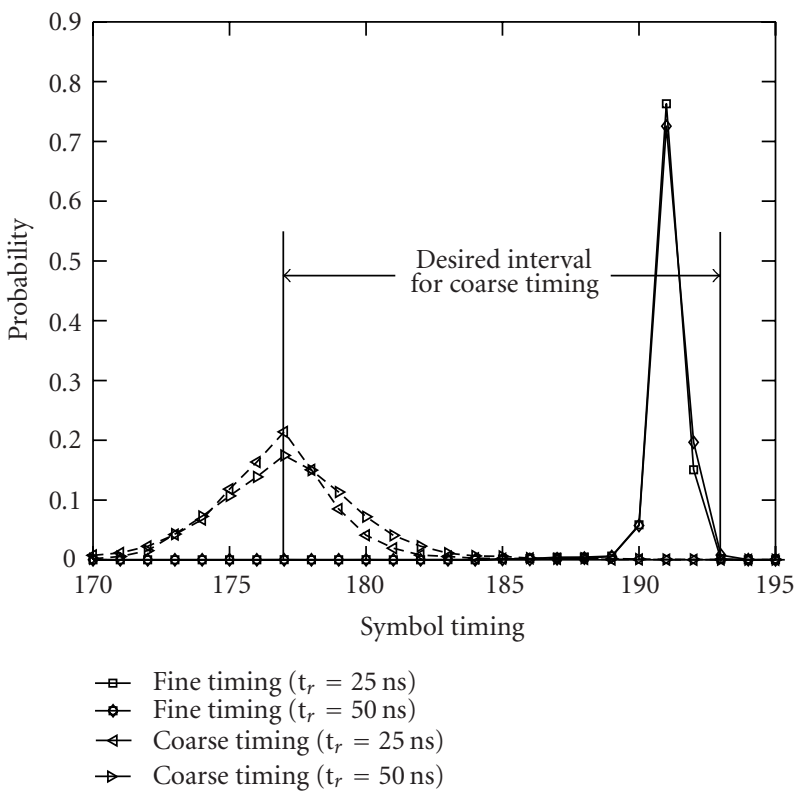

FIgUre 5: Coarse and fine symbol timing estimates.

put due to the input being the latter portion of the first half of GI2 is minimal. (Since the adverse effect of the coarse symbol timing estimate being larger than $T_{0}=193$ is usually troublesome for the fine CFO estimation, we prefer the coarse timing estimate $T_{C}$ to be well ahead of $T_{0}$.) The other two curves in Figure 5 show the $10^{4}$ Monte Carlo simulation results of the fine symbol timing estimates for the Chayat channels with $t_{r}=25$ and $t_{r}=50$ nanoseconds, respectively, when $\mathrm{SNR}=10 \mathrm{~dB}$. Note that our simple fine symbol timing approach gives highly accurate timing estimates.

We then provide a simulation example to show the effectiveness of the MIMO channel estimator and the PER performance of the MIMO soft detector. (One packet consists of 1000 bytes. Based on the IEEE 802.11a standard, even if only one error occurs in a packet, the entire packet is discarded.) In Figure 6, we show the $10^{4}$ Monte Carlo simulation results of the PER performance of our soft detector as a function of the SNR for the MIMO system, with $t_{r}$ being 50 nanoseconds for the Chayat channels, when the transmission data rate is $108 \mathrm{Mbps}$. We consider two cases: the case of perfect channel knowledge and the case of estimated channel parameters. For the former case, we assume the exact knowledge of $\mathrm{CFO}$, symbol timing, and MIMO channel, whereas for the latter case, we use the estimates of all of the aforementioned parameters obtained with our sequential approach from the MIMO packet preamble as well as the CFO residual phase tracking. As a reference, we also give the PER curves of the soft detector for the SISO system (with the data rate being $54 \mathrm{Mbps}$ ) as a reference. We note, from the PER curves, that both the MIMO preamble design and the sequential channel parameter estimation algorithm are effective in that the gap between the PER curves corresponding to the perfect channel knowledge case and the estimated channel parameter case for the MIMO system is no more than that of the SISO system. 


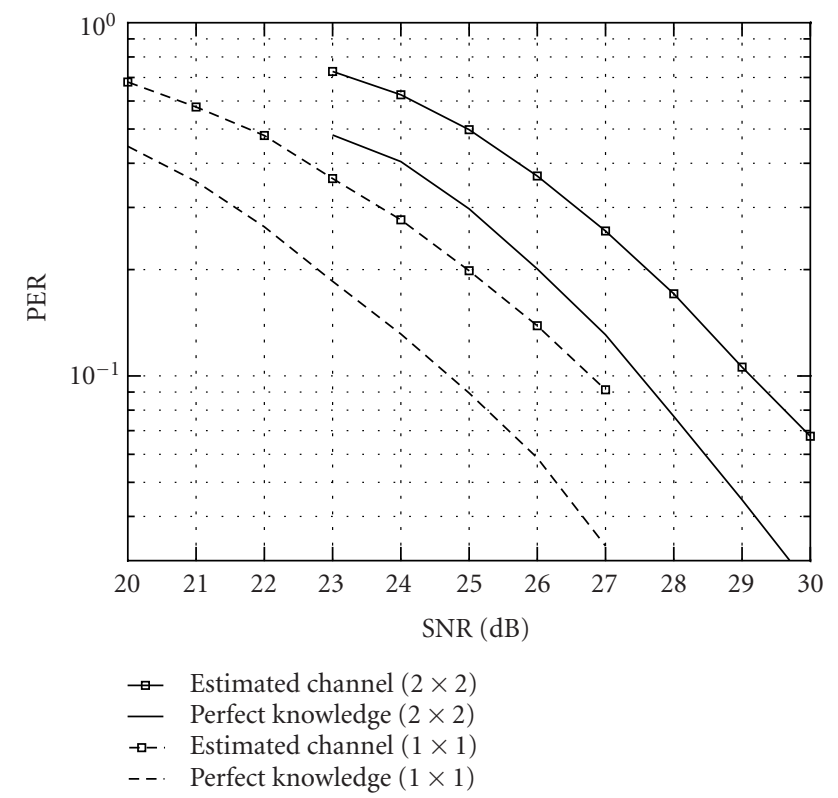

FIgURE 6: PER versus SNR at the $108 \mathrm{Mbps}$ data rate for the Chayat channels with $t_{r}=50$ nanoseconds.

Note also that the MIMO soft detector is effective in that the MIMO system needs only 2 to $3 \mathrm{~dB}$ extra total transmission power to keep the same PER (we are mostly interested in PERs being 0.1, according to the IEEE 802.11a standard) as its SISO counterpart, but with the data rate doubled.

Finally, we show the performance comparisons of the SPD hard detector [8], the MIMO soft detector, as well as the LSD-based soft detector [11], an approximation of STBICM, the ideal MIMO soft detector. Figure 7 gives PER curves obtained from $10^{4}$ Monte Carlo simulations for these detectors as a function of SNR for the MIMO system, with estimated channel parameters. (The simulation parameters are the same as those in the previous example.) Note that the MIMO soft detector is much better than SPD and is outperformed by LSD in terms of PER. However, the MIMO soft detector is much more efficient than LSD. We did not attempt to optimize our Matlab simulation codes. Even so, our preliminary results indicate that the LS-based MIMO soft detector requires only about one fifth of the computations needed by SPD. Unlike SPD, whose sphere radius shrinks when finding better solutions, LSD keeps the sphere radius constant, which means that it is computationally much more demanding than SPD, especially when the sphere radius is large. We do not have an exact flop comparison, yet we believe LSD flops $\gg$ SPD flops $\approx 5$ times MIMO soft detector flops, which means that the LS-based soft detector can be orders of magnitude more computationally efficient than the LSD-based one.

\section{CONCLUDING REMARKS}

We have proposed a preamble design for the MIMO system with two transmit and two receive antennas. This MIMO

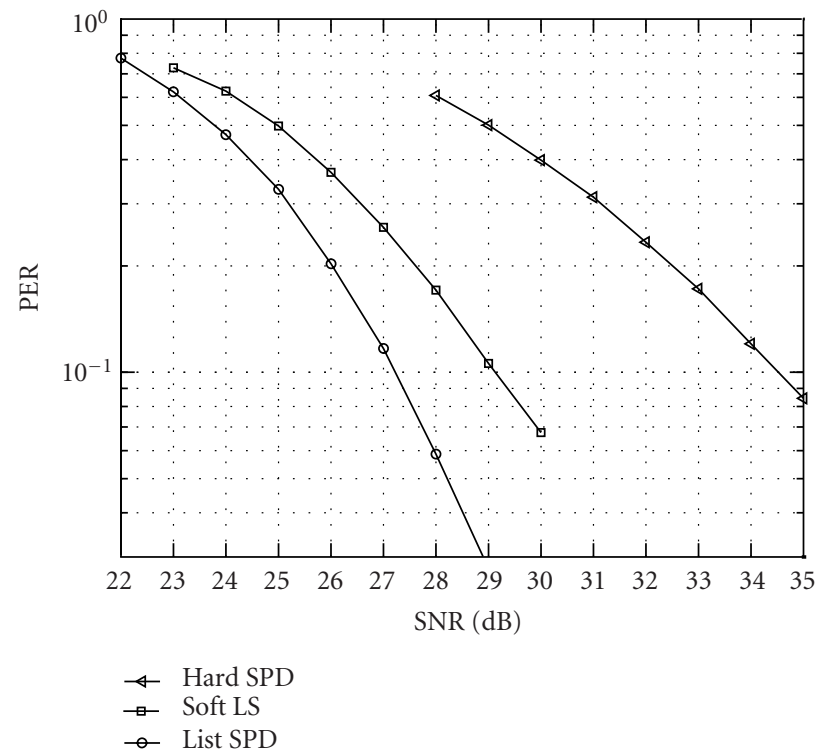

FIGURE 7: PER versus SNR at the $108 \mathrm{Mbps}$ data rate for the Chayat channels with $t_{r}=50$ nanoseconds.

preamble design is backward compatible with its SISO counterpart as specified by the IEEE 802.11a standard. Based on this MIMO preamble design, we have devised a sequential method for the estimation of CFO, symbol timing, and MIMO channel responses. We have also provided a simple soft detector for the MIMO system based on the unstructured LS approach to obtain the soft information for the Viterbi decoder. Both the sequential parameter estimation method and the soft detector are very efficient and ideally suited for real-time implementations. The effectiveness of our methods has been demonstrated via numerical examples.

\section{APPENDICES}

\section{A. BIT METRIC CALCULATION FOR THE QAM SYMBOL}

To make this paper self-contained, we describe in this appendix briefly our bit metric calculation method for the QAM symbol. Note that the real and imaginary parts of a QAM symbol plus the additive circularly symmetric complex Gaussian noise are independent of each other. Hence the soft decision statistic corresponding to a transmitted QAM symbol can be easily divided into the real and imaginary parts, which correspond to the soft decision statistic of two real valued PAM symbols. The variance of the noise additive to the PAM symbols is halved as compared to the QAM symbols. In view of this, we only present the method for calculating the bit metric for a symbol in the PAM constellation $\mathcal{R}$.

Let $\mathscr{D}_{i, j}=\{s: s \in \mathcal{R}\}$ denote the set of all the possible PAM symbols with the $i$ th bit $v_{i}=j, i=1,2, \ldots, \log _{2} A / 2$, $j=0,1$. The formation of $\mathcal{D}_{i, j}$ depends on the way the PAM symbols are labeled. For example, for the Gray indexed 8-ary 


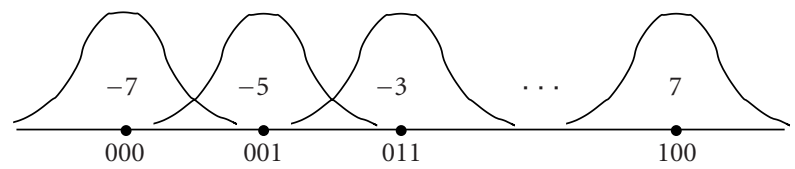

Figure 8: Illustration of 8-ary PAM symbols with Gray labeling and their pdf curves.

PAM constellation shown in Figure 8, we have

$$
\mathcal{D}_{1,0}=\{-7,-5,-3,-1\}
$$

where the first bit is the left most one shown in Figure 8. Then, for a given soft information $(x, \sigma)$ of the PAM symbol, the bit metric for $v_{i}$ is given by

$$
\hat{v}_{i}=\log \frac{p_{\sigma}\left(v_{i}=1 \mid x\right)}{p_{\sigma}\left(v_{i}=0 \mid x\right)},
$$

where

$$
\begin{aligned}
p_{\sigma}\left(v_{i}=j \mid x\right) & =p_{\sigma}\left(\mathcal{D}_{i, j} \mid x\right) \\
& =\sum_{s \in D_{i, j}} p_{\sigma}(s \mid x) \\
& =\sum_{s \in D_{i, j}} \frac{f_{\sigma}(x \mid s) p(s)}{p(x)}
\end{aligned}
$$

with

$$
f_{\sigma}(x \mid s)=\frac{1}{\sqrt{2 \pi} \sigma} e^{-(x-s)^{2} / 2 \sigma^{2}}
$$

being the pdf given the symbol $s$ and the variance $\sigma^{2}$, as shown in Figure 8. The occurrence of each symbol in $\mathcal{R}$ is often assumed to be equally likely, that is, $p(s)=(1 / 2)^{B / 2}$, for all $s \in \mathcal{R}$. In this case, we have

$$
p_{\sigma}\left(v_{i}=j \mid x\right)=\frac{1}{2^{B / 2} p(x)} \sum_{s \in D_{i, j}} f_{\sigma}(x \mid s),
$$

which leads to

$$
\hat{v}_{i}=\log \left\{\sum_{s \in \mathscr{D}_{i, 1}} e^{-(x-s)^{2} / 2 \sigma^{2}}\right\}-\log \left\{\sum_{s \in D_{i, 0}} e^{-(x-s)^{2} / 2 \sigma^{2}}\right\} .
$$

To speed up the bit metric calculation in practical applications, we can make a grid for $x$ and $\sigma$ to precalculate a lookup table for the $\hat{v}_{i}$ 's. The bit metric calculation in our simulations is based on such a table.

\section{B. A PROPERTY OF THE $c_{m}$ IN (33)}

With $\bar{x}$ and $\tilde{x}$ denoting the real and imaginary parts of $x$, respectively, we get from (33)

$$
\begin{aligned}
\mathbf{c} & =\left(\overline{\mathbf{H}}^{\dagger}+j \tilde{\mathbf{H}}^{\dagger}\right)(\overline{\mathbf{e}}+j \tilde{\mathbf{e}}) \\
& =\left(\overline{\mathbf{H}}^{\dagger} \overline{\mathbf{e}}-\tilde{\mathbf{H}}^{\dagger} \tilde{\mathbf{e}}\right)+j\left(\tilde{\mathbf{H}}^{\dagger} \overline{\mathbf{e}}+\overline{\mathbf{H}}^{\dagger} \tilde{\mathbf{e}}\right) .
\end{aligned}
$$

Then

$$
\overline{\mathbf{c}}=\overline{\mathbf{H}}^{\dagger} \overline{\mathbf{e}}-\tilde{\mathbf{H}}^{\dagger} \tilde{\mathbf{e}}, \quad \tilde{\mathbf{c}}=\tilde{\mathbf{H}}^{\dagger} \overline{\mathbf{e}}+\overline{\mathbf{H}}^{\dagger} \tilde{\mathbf{e}}
$$

Hence, we have

$$
\begin{aligned}
\mathrm{E}\left[\overline{\mathbf{c}} \tilde{\mathbf{c}}^{T}\right]= & \mathrm{E}\left[\left(\overline{\mathbf{H}}^{\dagger} \overline{\mathbf{e}}-\tilde{\mathbf{H}}^{\dagger} \tilde{\mathbf{e}}\right)\left(\overline{\mathbf{e}}^{T}\left(\tilde{\mathbf{H}}^{\dagger}\right)^{T}+\tilde{\mathbf{e}}^{T}\left(\overline{\mathbf{H}}^{\dagger}\right)^{T}\right)\right] \\
= & \mathrm{E}\left[\overline{\mathbf{H}}^{\dagger} \overline{\mathbf{e}} \overline{\mathbf{e}}^{T}\left(\tilde{\mathbf{H}}^{\dagger}\right)^{T}-\tilde{\mathbf{H}}^{\dagger} \tilde{\mathbf{e}} \tilde{\mathbf{e}}^{T}\left(\overline{\mathbf{H}}^{\dagger}\right)^{T}\right] \\
& +\mathrm{E}\left[\overline{\mathbf{H}}^{\dagger} \overline{\mathbf{e}} \tilde{\mathbf{e}}^{T}\left(\overline{\mathbf{H}}^{\dagger}\right)^{T}-\tilde{\mathbf{H}}^{\dagger} \tilde{\mathbf{e}}^{T}\left(\tilde{\mathbf{H}}^{\dagger}\right)^{T}\right] \\
= & \frac{1}{2} \sigma^{2}\left[\overline{\mathbf{H}}^{\dagger}\left(\tilde{\mathbf{H}}^{\dagger}\right)^{T}-\tilde{\mathbf{H}}^{\dagger}\left(\overline{\mathbf{H}}^{\dagger}\right)^{T}\right] \\
= & \frac{1}{2} \sigma^{2}\left[\overline{\mathbf{H}}^{\dagger}\left(\tilde{\mathbf{H}}^{\dagger}\right)^{T}-\left(\overline{\mathbf{H}}^{\dagger}\left(\tilde{\mathbf{H}}^{\dagger}\right)^{T}\right)^{T}\right],
\end{aligned}
$$

where we have used the fact that $\mathrm{E}\left[\overline{\mathbf{e}} \overline{\mathbf{e}}^{T}\right]=\mathrm{E}\left[\tilde{\mathbf{e}} \tilde{\mathbf{e}}^{T}\right]=\sigma^{2} \mathbf{I} / 2$ and $\mathrm{E}\left[\overline{\mathbf{e}} \tilde{\mathbf{e}}^{T}\right]=\mathrm{E}\left[\tilde{\mathbf{e}} \overline{\mathbf{e}}^{T}\right]=\mathbf{0}$. Equation (B.3) implies that the diagonal elements of $\mathrm{E}\left[\overline{\overline{\mathbf{c}}} \tilde{\mathbf{c}}^{T}\right]$ are zero and hence $\mathrm{E}\left[\overline{\boldsymbol{c}}_{m} \tilde{c}_{m}\right]=0$, $m=1,2, \ldots, M$.

\section{PHASE CORRECTION USING PILOT SYMBOLS}

The CFO correction will never be perfect in practice due to the presence of noise. Hence, there will be a phase error $\phi$ for each OFDM data symbol caused by the error in the fine CFO estimate $\hat{\epsilon}_{F}$. The error $\phi$ increases linearly with time.

As we mentioned earlier, each OFDM data symbol contains four known pilot symbols. We denote these pilot symbols by a $4 \times 1$ vector $\mathrm{p}$. The pilot symbols can be used to correct $\phi$ for each OFDM data symbol after CFO correction using $\hat{\epsilon}_{F}$. Let $\mathbf{y}_{n}^{(\mathrm{p})}$ be the vector containing the corresponding four elements of the FFT output of an OFDM data symbol in the OFDM data field received from the $n$th antenna, $n=1,2, \ldots, N$. Let $\hat{\mathbf{h}}_{n, m}^{(\mathrm{p})}$ be the $4 \times 1$ estimated channel vector from transmit antenna $m$ to receive antenna $n$ for the four corresponding subcarriers. Let $\mathbf{P}=\operatorname{diag}\{\mathbf{p}\}$. We have

$$
\mathbf{y}_{n}^{(\mathrm{p})}=e^{j \phi} \mathbf{P} \sum_{m=1}^{2} \hat{\mathbf{h}}_{n, m}^{(\mathrm{p})}+\mathbf{e}_{n}^{(\mathrm{p})}, \quad n=1,2, \ldots, N,
$$

where $\left\{\mathbf{e}_{n}^{(\mathrm{p})}\right\}_{n=1}^{N}$ are zero-mean white circularly symmetric complex Gaussian noise vectors that are independently and identically distributed. Then the ML criterion leads to

$$
\begin{aligned}
\hat{\phi}_{\mathrm{ML}} & =\arg \min _{\phi} \sum_{n=1}^{N}\left\|\mathbf{y}_{n}^{(\mathrm{p})}-e^{j \phi} \mathbf{P} \sum_{m=1}^{2} \hat{\mathbf{h}}_{n, m}^{(\mathrm{p})}\right\|^{2} \\
& =\angle\left\{\sum_{n=1}^{N}\left[\sum_{m=1}^{2}\left(\hat{\mathbf{h}}_{n, m}^{(\mathrm{p})}\right)^{H}\right] \mathbf{P}^{H} \mathbf{y}_{n}^{(\mathrm{p})}\right\} .
\end{aligned}
$$

\section{ACKNOWLEDGMENT}

This work was supported in part by the National Science Foundation Grant CCR-0097114 and the Intersil Corporation Contract 2001056. 


\section{REFERENCES}

[1] R. van Nee, G. Awater, M. Morikura, H. Takanashi, M. Webster, and K. W. Halford, "New high-rate wireless LAN standards," IEEE Communications Magazine, vol. 37, no. 12, pp. 82-88, 1999.

[2] IEEE 802.11a-1999, "IEEE Standard for Information technology-Telecommunications and information exchange between systems-Local and metropolitan area networksspecific requirements-Part 11: Wireless LAN Medium Access Control (MAC) and Physical Layer (PHY) specifications-Amendment 1: High-speed Physical Layer in the 5 GHz band," 1999.

[3] A. F. Naguib, N. Seshadri, and A. R. Calderbank, "Increasing data rate over wireless channels," IEEE Signal Processing Magazine, vol. 17, no. 3, pp. 76-92, 2000.

[4] G. J. Foschini, "Layered space-time architecture for wireless communication in a fading environment when using multielement antennas," Bell Labs Tech. Journal, vol. 1, no. 2, pp. 41-59, 1996.

[5] G. D. Golden, G. J. Foschini, R. A. Valenzuela, and P. W. Wolniansky, "Detection algorithm and initial laboratory results using the V-BLAST space-time communication architecture," Electronics Letters, vol. 35, no. 1, pp. 14-15, 1999.

[6] G. J. Foschini and M. J. Gans, "On limits of wireless communications in a fading environment when using multiple antennas," Wireless Personal Communications, vol. 6, no. 3, pp. 311-335, 1998.

[7] I. E. Telatar, "Capacity of multi-antenna Gaussian channels," European Transactions on Telecommunications, vol. 10, no. 6, pp. 585-595, 1999.

[8] O. Damen, A. Chkeif, and J.-C. Belfiore, "Lattice code decoder for space-time codes," IEEE Communications Letters, vol. 4, no. 5, pp. 161-163, 2000.

[9] X. Li, H. Huang, G. J. Foschini, and R. A. Valenzuela, "Effects of iterative detection and decoding on the performance of BLAST," in Proc. IEEE GLOBECOM'00, vol. 2, pp. 10611066, San Francisco, Calif, USA, November 2000.

[10] A. M. Tonello, "Space-time bit-interleaved coded modulation with an iterative decoding strategy," in Proc. IEEE 52th Vehicular Technology Conference, vol. 1, pp. 473-478, Boston, Mass, USA, September 2000.

[11] B. M. Hochwald and S. ten Brink, "Achieving near-capacity on a multiple-antenna channel," IEEE Transactions on Communications, vol. 51, no. 3, pp. 389-399, 2003.

[12] C. Z. W. Hassell Sweatman, J. S. Thompson, B. Mulgrew, and P. M. Grant, "A comparison of the MMSE detector and its BLAST version for the MIMO channel," in IEE Seminar on MIMO: Communications Systems from Concept to Implementations, pp. 19/1-19/6, London, UK, December 2001.

[13] J.-J. van de Beek, O. Edfors, M. Sandell, S. K. Wilson, and P. O. Börjesson, "On channel estimation in OFDM systems," in Proc. IEEE 45th Vehicular Technology Conference, vol. 2, pp. 815-819, Chicago, Ill, USA, July 1995.

[14] Z. Wang and G. B. Giannakis, "Wireless multicarrier communications," IEEE Signal Processing Magazine, vol. 17, pp. 29-48, May 2000.

[15] N. Chayat, "Tentative criteria for comparison of modulation methods," IEEE P802.11-97/96, September 1997.

[16] E. G. Larsson and J. Li, "Preamble design for multipleantenna OFDM-based WLANs with null subcarriers," IEEE Signal Processing Letters, vol. 8, no. 11, pp. 285-288, 2001.

[17] T. M. Schmidl and D. C. Cox, "Robust frequency and timing synchronization for OFDM," IEEE Transactions on Communications, vol. 45, no. 12, pp. 1613-1621, 1997.
[18] J. Li, G. Liu, and G. B. Giannakis, "Carrier frequency offset estimation for OFDM-based WLANs," IEEE Signal Processing Letters, vol. 8, no. 3, pp. 80-82, 2001.

Jianhua Liu received the B.S. degree in electrical engineering from Dalian Maritime University, Dalian, China, in 1984, the M.S. degree in electrical engineering from the University of Electronic Science and Technology of China, Chengdu, China, in 1987, and the Ph.D. degree in electronic engineering from Tsinghua University, Beijing, China, in 1998. From March 1987 to February 1999, he worked at the Communica-

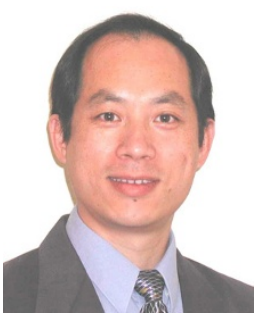
tions, Telemetry, and Telecontrol Research Institute, Shijiazhuang, China, where he was an Assistant Engineer, Engineer, Senior Engineer, and Fellow Engineer. From March 1995 to August 1998, he was also a Research Assistant at Tsinghua University. From February 1999 to June 2000, he worked at Nanyang Technological University, Singapore, as a Research Fellow. Since June 2000, he has been a Research Assistant in the Department of Electrical and Computer Engineering, the University of Florida, Gainesville, working towards a Ph.D. degree majoring in electrical engineering and minoring in Statistics. His research interests include wireless communications, statistical signal processing, and sensor array processing.

Jian Li received the M.S. and Ph.D. degrees in electrical engineering from The Ohio State University, Columbus, in 1987 and 1991, respectively. From April 1991 to June 1991, she was an Adjunct Assistant Professor with the Department of Electrical Engineering, The Ohio State University, Columbus. From July 1991 to June 1993, she was an Assistant Professor with the Department of Electrical Engineering, University of Ken-

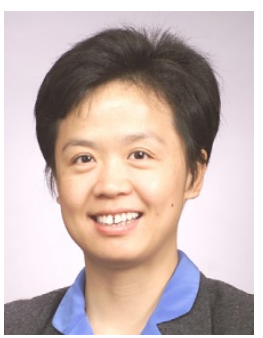
tucky, Lexington. Since August 1993, she has been with the Department of Electrical and Computer Engineering, University of Florida, Gainesville, where she is currently a Professor. Her current research interests include spectral estimation, array signal processing, and their applications. Dr. Li is a member of Sigma Xi and Phi Kappa Phi. She received the 1994 National Science Foundation Young Investigator Award and the 1996 Office of Naval Research Young Investigator Award. She was an Executive Committee Member of the 2002 International Conference on Acoustics, Speech, and Signal Processing, Orlando, Florida, May 2002. She has been an Associate Editor of the IEEE Transactions on Signal Processing since 1999 and an Associate Editor of the IEEE Signal Processing Magazine since 2003. She is presently a member of the Signal Processing Theory and Methods (SPTM) Technical Committee of the IEEE Signal Processing Society. 